\section{Learning in order}

According to a new study by Pasupathy and Miller, the neural correlates of associative learning appear in the caudate nucleus - part of the basal ganglia - earlier than in the prefrontal cortex (PFC). The findings shed new light on the interactions and relationships between the basal ganglia and cortex.

The authors used a simple conditional association learning task to investigate the roles of the two brain areas. Monkeys had to learn to make a leftward eye movement in response to one visual cue, and a rightward eye movement in response to another. Correct responses were rewarded with fruit juice, and once a pair of cues had been learned the required responses were reversed so that the monkeys had to learn the new associations. New cues were used each day, and Pasupathy and Miller recorded the activity of neurons in both the dorsolateral PFC and the caudate nucleus during the task.

Both the dorsolateral PFC and the caudate nucleus are known to show task-related activity in this sort of task, and they are joined as part of a corticostriatal loop, but until now the temporal relationship between their activity has not been

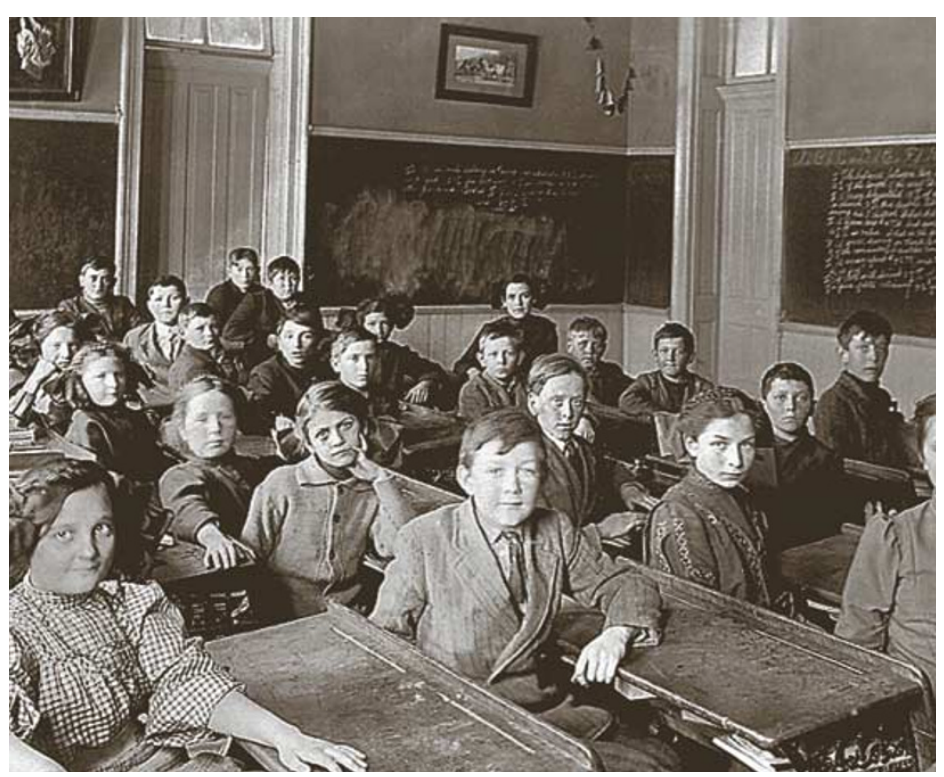

investigated. The authors found that neurons in both areas showed changes in activity during associative learning, but that the neurons in the caudate nucleus showed these changes much earlier, and more abruptly, than did neurons in the dorsolateral PFC. idea that the basal ganglia can learn new associations rapidly, and then 'train' the PFC. Interestingly, the monkeys' behaviour showed slower learning of the new cue-response associations than did the activity of caudate neurons. Whereas caudate activity showed a sudden switch to the correct association, the monkeys' performance improved more gradually, and was correlated more strongly with the changes in activity seen in the PFC. The authors suggest that although the caudate nucleus might 'train' the PFC, behaviour depends more on PFC activity than on that of the basal ganglia.

Rachel Jones

(1) References and links ORIGINAL RESEARCH PAPER Pasupathy, A. \& Miller, E. K. Different time courses of learningrelated activity in the prefrontal cortex and striatum. Nature 433, 873-876 (2005)

WEB SITE

Miller's laboratory: http://www.millerlab.org/
These results might support the

\section{IN BRIEF}

GENES AND DISEASE

Pharmacological rescue of synaptic plasticity, courtship behavior, and mushroom body defects in a Drosophila model of fragile $X$ syndrome.

McBride, S. M. J. et al. Neuron 45, 753-764 (2005)

In humans, the loss of FMR1 gene function, which causes fragile $\mathrm{X}$ syndrome, is the leading cause of inherited mental retardation. Loss of function of the homologous gene in fruit flies produces a model of the syndrome, with abnormalities in circadian and courtship behaviour and neuroanatomical changes. In addition, McBride et al. find that the flies show impaired learning. The authors show that treatment with antagonists of metabotropic glutamate receptors or with lithium can rescue the behavioural, neuroanatomical and cognitive abnormalities in the fly model. The pharmacological rescue of the symptoms indicates that it might be possible to treat patients with fragile $\mathrm{X}$ syndrome using a similar approach.

\section{BRAIN EVOLUTION}

\section{The brain of LB1, Homo floresiensis.}

Falk, D. et al. Science 3 March 2005 (10.1126/science.1109727)

Homo floresiensis was a diminutive species of human nicknamed 'hobbits' by their discoverers - that lived about $18,000-38,000$ years ago on the island of Flores in Indonesia. Falk and colleagues have assessed the brain of $H$. floresiensis by studying a three-dimensional reconstruction based on the skull of the type specimen, LB1. Comparisons with endocasts from the skulls of apes, Homo erectus, Homo sapiens, Australopithecus africanus and Paranthropus aeithiopicus indicate that although LB1's brain/body size ratio is similar to that of A. africanus, the shape is more similar to that of $H$. erectus, but with an expanded temporal lobe. The authors suggest that, despite its small brain, $H$. floresiensis was capable of relatively advanced cognition.

\section{AXON GUIDANCE}

$\beta 1,3-N$-Acetylglucosaminyltransferase 1 glycosylation is required for axon pathfinding by olfactory sensory neurons.

Henion, T. R. et al. J. Neurosci. 25, 1894-1903 (2005)

Axonal pathfinding in the embryonic olfactory system enables olfactory sensory neurons to send their axons to specific glomeruli in the olfactory bulb, so that each glomerulus receives inputs from neurons that express the same odorant receptor. The odorant receptors themselves, along with other guidance factors, have been implicated in axonal pathfinding, but the mechanism by which this precision is achieved remains unclear. Henion et al. now show that glycans are also crucial for precise axonal pathfinding in the olfactory system - mice that lack the glycosyltransferase $\beta 1,3$ - $N$-acetylglucosaminyltransferase 1 have abnormal olfactory bulb innervation and impaired formation of glomeruli in the olfactory bulb. 